\title{
La formación didáctica en educación física del docente de básica primaria en escuelas colombiana
}

\author{
The didactic training in physical education of the elementary school teacher in \\ Colombian schools \\ A formação didática em educação física do professor do ensino fundamental nas escolas \\ colombianas
}

ARTÍCULO DE REVISIÓN

\author{
Carlos Palacios \\ capalo24@ hotmail.com \\ Sunny R. Perozo Ch. \\ sunperozo@gmail.com \\ ORCID: 0000-0002-1140-5826 \\ ORCID: 0000-0001-7574-1995
}

Universidad Metropolitana Educación Ciencia y Tecnología, Panamá

Recibido abril 2020 | Revisado mayo 2020 | Publicado en julio 2020

\section{RESUMEN}

El propósito de este trabajo fue profundizar en la necesidad de la formación didáctica en educación física de los docentes de la básica primaria en las escuelas colombianas; para lo cual se realizó una revisión teórica conceptual de los aspectos básicos del manejo de la educación física para los niños de primaria y sus beneficios a nivel de desarrollo corporal y emocional. Metodológicamente se utilizó la revisión documental basada en los niveles monográficos $\mathrm{y}$ hermenéuticos como técnicas para la revisión y análisis de fuentes bibliográficas. Los resultados inducen a afirmar que los docentes de la básica primaria de las escuelas colombianas no poseen la formación didáctica en educación física para desarrollar procesos de enseñanza acordes con las características de la asignatura, lo cual limita los beneficios biopsicosociales que deberían ser adquiridos durante la formación del niño en esta etapa escolar.

Palabras clave: Educación física; beneficio; eficacia, flexibilidad

\begin{abstract}
The purpose of this work was to deepen the need for didactic training in physical education for elementary school teachers in Colombian schools; for which a conceptual theoretical review of the basic aspects of physical education management for primary school children and its benefits at the level of body and emotional development was carried out. Methodologically, the documentary review based on the monographic and hermeneutical levels was used as techniques for the review and analysis of bibliographic sources. The results lead to affirm that the teachers of elementary school in Colombian schools do not have the didactic training in physical education to develop teaching processes in accordance with the characteristics of the subject, which limits the biopsychosocial benefits that should be acquired during training of the child in this school stage.
\end{abstract}

Key words: Physical education; benefit; efficiency, flexibility 


\section{RESUMO}

O objetivo deste trabalho foi aprofundar a necessidade de formação didática em educação física para professores do ensino fundamental em escolas colombianas; para o qual foi realizada uma revisão teórica conceitual dos aspectos básicos da gestão da educação física para crianças do ensino fundamental e seus benefícios ao nível do desenvolvimento corporal e emocional. Metodologicamente, a revisão documental baseada nos níveis monográfico e hermenêutico foi utilizada como técnica para a revisão e análise de fontes bibliográficas. Os resultados levam a afirmar que os professores do ensino fundamental nas escolas colombianas não possuem a formação didática em educação física para desenvolver processos de ensino de acordo com as características da disciplina, o que limita os benefícios biopsicossociais que devem ser adquiridos durante a formação. Da criança nesta fase escolar.

Palavras-chave: Educação física; beneficiar; eficiência, flexibilidade

\section{INTRODUCCIÓN}

En las últimas décadas, el mundo ha evolucionado más que en los dos siglos anteriores. El ritmo acelerado de la vida cotidiana, la modificación de las relaciones entre hombres y mujeres, debida en buena medida a la incorporación de la mujer al mundo laboral, la mundialización y el acceso cada vez mayor a los medios de comunicación, han contribuido a una simplificación de los usos tradicionales. Muchas reglas, aun cuando todavía moderen ciertas situaciones en determinados contextos, tienden a desaparecer al convertirse en inútiles, molestas o simplemente anacrónicas.

La escuela y el docente del siglo XXI no escapan a esta realidad y su autenticidad y utilidad sólo se hará realidad en la medida en que puedan asumir los retos que ofrece el mundo cambiante de hoy donde las nuevas maneras de enseñar y aprender están signadas por la transformación y los cambios, lo que requiere que el docente de estos tiempos actualice su cosmovisión de gestor de aprendizaje a un concepto más dinámico que le permita trascender de lo eminentemente académico-tradicional hacia una concepción educativa holística donde al estudiante se le reconozca como un ser biopsicosocial con potencialidades que afianzan su autoconocimiento, factor que guía la construcción que haga de las bases para su vida y de las soluciones que aporte a las problemáticas sociales de su entorno.

La educación como principal medio de socialización propicia la formación integral de las capacidades volitivas, afectivas y biopsicosociales del ser humano; es en esta realidad donde la acción de la escuela y del maestro tienen protagonismo estelar mediante la oportunidad que abre de establecer acciones pedagógicas orientadas a desarrollar y fortalecer en cada persona, las competencias de aprendizaje para el saber hacer, el saber conocer y el saber ser, en todas las áreas del conocimiento, como bases fundamentales para el desenvolvimiento pleno dentro de su contexto.

Conocer el origen y evolución de la disciplina encerrada en los programas de educación física es de gran relevancia para quien pretende dar a conocer la importancia que tiene en el éxito personal el mantenerse físicamente sano, comer razonablemente, conocer las propias limitaciones, así como ejercer control sobre las preocupaciones y temores por medio de los efectos del deporte, hay que recordar el viejo adagio: "sólo consumiendo el producto...puede venderse".

\section{MÉTODO}

Este articulo muestra la orientación que tuvo el estudio teórico conceptual sobre la formación didáctica de los docentes de educación física, para lo cual se realizó una 
documentación mediante el desarrollo de nivel monográfico relacionado con la descripción temática del material planteado por los autores, sin que el investigador asuma alguna postura o posición particular (Hurtado 2010). De esta manera, se plantea el uso de la técnica de Riaceer la cual comprende la recopilación, identificación de ideas, almacenamiento, categorización, esquema conceptual y redacción del documento), ante lo cual se revisaron los diferentes aspectos conceptuales planteados por los autores sobre el tema estudiado. El otro nivel fue el hermenéutico que se realizó mediante una interpretación de los aspectos conceptuales presentados en el nivel monográfico a partir del entendimiento, sustentación, alcance y vigencia de los planteamientos (Hurtado, 2010).

\section{DESARROLLO Y DISCUSIÓN}

Sin duda alguna, la educación es de gran importancia, no solo para el desarrollo individual sino también social. En cuanto más educados (crianza, enseñanza o instrucción), mayor posibilidad de mejorar la capacidad de relación con los demás; mejor prestación de servicios a las comunidades y mejores remuneraciones por servicio o desempeño laboral, es decir, la educación está en relación directa con la calidad de vida de la sociedad.

A través de la educación, las generaciones no solo asimilan y aprenden conocimientos, normas de conducta y modos de ser, sino también se llega a conocer las otras formas en que las generaciones anteriores vivían y veían el mundo, para así al crear en el presente un mundo nuevo, evitar cometer los errores y horrores del pasado.

Pese a que los expertos tienen muy claro, y muchos concuerdan en cuál es la importancia de la educación y sus alcances, se han generado muchas disyuntivas en cuanto a su definición.
Una de las definiciones más significativas, por apuntar no solo a lo cognitivo sino también a lo social y moral, es la de Valenzuela (2010), “...es un proceso en donde se transmiten conocimientos, valores, costumbres, comportamientos, actitudes y formas de actuar...; todo ello para poder desenvolvernos de manera activa y participativa en sociedad". (p. 33) Al respecto, Batista (2006) afirma, "la educación posibilita la adquisición de conocimientos y saberes; la instrucción está ligada a la calidad de vida de las personas y sociedades, como también lo está la salud pública para quienes son social, política y jurídicamente" (p. 28).

Se observa que estos autores coinciden en definir la educación como un proceso que conlleva a que las personas desarrollen sus capacidades intelectuales mediante el logro de conocimientos $\mathrm{y}$, por consiguiente, esto permite mejorar su calidad de vida, y la de las sociedades y contribuye a la igualdad social, donde cada persona tiene la posibilidad de participar en la transformación de realidades sociales, lo cual favorece el desarrollo social, la igualdad, la participación activa y la toma de decisiones.

De esta manera, la educación es reconocida como un proceso de socialización de las personas, al permitir la interacción de los grupos y asimilar y adquirir un conocimiento para el desarrollo de habilidades sociales que conllevan a aprender a convivir. Es por esto que el proceso educativo, se materializa en habilidades y valores que son capaces de producir variantes de carácter emocional, intelectual, moral y social en las personas.

$\mathrm{Al}$ respecto Henz (1976) expone, "la educación es el conjunto de todos los efectos procedentes de personas, de sus actividades y actos...que resultan 'beneficiosos' para el individuo, despertando y fortaleciendo en él sus capacidades esenciales para que pueda... 
participar responsablemente en la sociedad...". (p. 39) Esto implica que una buena educación genera transformaciones positivas en el individuo y lo prepara no solo para ir en busca de su propia felicidad, sino también lo capacita para alcanzar la felicidad del colectivo mediante una convivencia armoniosa y pacífica con sus congéneres y con la naturaleza.

En concordancia con Henz (1976), autores como Castillo y Gamboa (2012), afirman que la educación "es un proceso por medio del cual el ser humano adquiere distintas herramientas para su inserción en la sociedad y su realización personal". En este proceso, claro está, intervienen distintas disciplinas con el propósito de facilitar su comprensión, guía y estudio.

En América Latina la educación, según lo expresa la Unesco (2014), pasa por tres aspectos relativamente críticos. El primer aspecto está referido a los logros, los cuales no se replican en todos los países, pues las diferencias al interior de las regiones son marcadas y varios países están muy lejos, incluso de las metas básicas de Dakar orientadas a cumplir los objetivos que permitan una educación para todos; obligación de los gobiernos nacionales de velar por el alcance de los objetivos y finalidades de la educación para todos; y que todos los niños, jóvenes y adultos, tienen derecho a una educación que satisfaga sus necesidades básicas de aprendizaje y que permita asimilar conocimientos, a hacer, a vivir con los demás y a ser. En síntesis, se pretende que los niños y adolescentes a nivel mundial cumplan los estándares mínimos de calidad en áreas como de lectura, matemáticas y convivencia.

En este sentido, es necesario mencionar que más de la mitad de niños y adolescentes a nivel mundial no cumplen los estándares mínimos en las áreas de lectura y matemáticas. Hay disparidad en la educación a niveles de género, ubicación (rural o urbana) y otros aspectos. Se requiere más inversión en la educación, sobre todo en los países en vía de desarrollo. La tasa de analfabetismo adulto es alta, obviamente como resultado de estas disparidades, lo cual es muy lamentable.

La educación es un derecho humano básico, todas las personas, con independencia de su género, origen étnico o situación económica, tienen derecho a ella. Sin embargo, esto solo se da cuando gobiernos, ciudadanos, organizaciones de desarrollo y medios de comunicación colaboran con el fin de ayudar a todos los niños y niñas de todos los países del mundo, y de esta manera exigen (u obligan) a que verdaderamente se les respete el derecho inalienable a una educación de calidad. Pero para ello, se necesitan metas claras y mensurables, acompañadas de indicadores que permitan efectuar un seguimiento de los progresos de los más desfavorecidos.

Por otra parte, los gobiernos a través de sus ministerios y secretarías de educación, permanentemente deben hacer acompañamiento, vigilancia, seguimiento $\mathrm{y}$ control para asegurarse de que todos los niños estén escolarizados y adquieran los conocimientos básicos. Los niños y niñas no solo tienen derecho a estar escolarizados, sino también a aprender mientras asisten a la escuela y a contar con las competencias que necesitan para encontrar un trabajo bien pagado y seguro cuando terminan sus estudios.

De igual manera, los gobiernos deben velar por que los mejores docentes puedan enseñar a los estudiantes que más los necesitan. Los planes nacionales de educación 
deben incluir el compromiso expreso de atender a los marginados. Deben contratarse a los docentes localmente o bien, asegurarse de que tengan orígenes similares a los de los estudiantes desfavorecidos.

Se debe, además, proporcionar incentivos con objeto de garantizar que los mejores docentes trabajen en las zonas remotas y menos atendidas. Los gobiernos han de conservar a sus mejores docentes por medio de un salario que satisfaga por lo menos sus necesidades básicas así como unas buenas condiciones de trabajo y una trayectoria profesional estructurada.

El segundo aspecto se refiere a las desigualdades internas, las cuales son muy agudas en prácticamente todos los países de América Latina, siendo la clase social, la condición indígena y la zona de residencia, los vectores principales de estas desigualdades. En nuestro país, por ejemplo, según los resultados de las pruebas SABER, año 2017, la mayoría de niños y adolescentes no cumplen los estándares mínimos de competencias en las áreas de lectura y matemáticas. Hay disparidad en la educación a nivel de lo público y lo privado; la ubicación (rural o urbana) y otros aspectos. Se requiere más inversión en la educación, sobre todo en las zonas o departamentos en donde la calidad de la educación es muy baja, obviamente como resultado de estas disparidades.

Si hay un indicador que representa el nivel de desarrollo de un país es, sin duda, el que se refiere a la educación. A pesar del reconocimiento que todos los países parecen dar al peso de la educación, la desigualdad en el acceso a la escuela primaria y la necesaria inversión en la mejora de la calidad educativa acrecienta la brecha entre clases sociales y entre países ricos y países pobres. Mientras que para estos últimos se hacen intermitentes y pocos metódicos esfuerzos para garantizar el acceso a la escuela para todos sus ciudadanos y poder asegurarles una mínima educación básica, los países desarrollados invierten cada vez más en la formación terciaria y en la mejora de la formación de los profesores.

Un informe hecho por la Unesco en el año 2016 sobre el seguimiento de la educación mundial mostró el fracaso de la comunidad internacional en su intento de abordar la pobreza y la desigualdad en el mundo. Según la Unesco lo establecido en los objetivos de desarrollo del milenio son imposibles de alcanzar sin un verdadero compromiso de los gobierno con unas políticas claras y reales en el marco de la educación igualitaria; por la misma razón, es probable que tampoco se cumplan los propuestos para el año 2030, que son mucho más ambiciosos. La desigualdad económica y social, los conflictos armados y las diferencias políticas son otros grandes y principales obstáculos. La cambiante realidad del mundo global requiere también incluir la educación para la democracia y la paz entre los objetivos prioritarios si se quiere abordar una educación verdaderamente de calidad.

Por último, cada vez más los nuevos criterios con que se deben juzgar los avances en educación remiten a la calidad más que a la simple expansión de la educación. La concepción de calidad es amplia e incluye no solo logros, sino condiciones y procesos, no solo aspectos académicos, sino sicosociales y ciudadanos. La calidad, es sin duda el aspecto en el que la región de América Latina se encuentra crónicamente más retrasada.

Con referencia a la calidad, expresa la Unesco (2013) que, aunque ésta depende de múltiples factores, son en última, los logros, las condiciones y procesos, los aspectos académicos, sociales y ciudadanos, los que definen a la educación como de buena o mala calidad. 
Es filosóficamente atípico que el desarrollo de una sociedad no esté subordinado a la calidad de la educación que reciben sus individuos. Un pueblo que no esté bien educado no planifica su futuro.

Según la Unesco (2011), la educación de calidad debe llegar a ser una realidad para todos, de modo que cada individuo disponga de la oportunidad de hacer realidad todo su potencial y disfrutar de mejor salud, mejorar sus condiciones de vida y lograr una participación social y política más plena en la vida colectiva. Así, al menos en teoría, las personas podrían mejorar su calidad de vida.

De esta manera, en Colombia, la Constitución Política y la Ley General de Educación (Ley 115), expresan el compromiso de garantizar una educación de calidad en todos los niveles de enseñanza. Es por ello, que el estado no sólo traza líneas generales sobre el tipo de hombre que desea formar y los fines y objetivos de la educación, sino también brinda las herramientas, condiciones, mecanismos y recursos para que se pueda ofrecer una educación con calidad y de calidad.

Sin embargo, para que la educación de calidad pueda lograrse, es necesario que en el proceso educativo, tanto maestros como estudiantes tengan $\mathrm{y}$ desarrollen roles definidos claramente en el proceso de enseñanza aprendizaje. De igual manera, se requiere que el maestro tenga la habilidad de crear en el aula una atmósfera que invite a todos a aprender, a construir el aprendizaje.

En consecuencia, cumplir el rol de docente involucra no solo poner en juego su formación pedagógica al proporcionar información y controlar la disciplina, sino -y sobre todo-, ser un mediador entre el alumno y el ambiente, lo que implica dejar de ser el protagonista del aprendizaje para pasar al rol de guía o acompañante del alumno en su proceso de aprehensión del conocimiento.

Por su parte, el estudiante debe ser reflexivo, tener iniciativa en la resolución de problemas, ser capaz de tomar decisiones, identificar relaciones entre teoría y práctica, ser crítico, propositivo y, sobre todo, tener vocación autodidacta. Dicho de otro modo, debe ser un sujeto activo, que investiga, promueve y es protagonista de su propio aprendizaje.

Actualmente la educación plantea unos propósitos referidos a la formación pedagógica y didáctica de quienes tienen la responsabilidad de desarrollar el proceso educativo. Por tanto, el docente de hoy está obligado a sufrir una especie de "metamorfosis ideológica" para pasar de ser un operador de currículos, reproductor de teorías y conceptos, a convertirse en un investigador permanente y autónomo del hecho pedagógico.

Según De Zubiría (2018), los maestros, se cuentan entre los profesionales que alcanzan los más bajos niveles en lectura crítica y escritura, lo que permite afirmar que la calidad de la educación, sólo cambiará cuando cambie este aspecto; situación que remite a la necesidad imperiosa de que el docente se preocupe y se ocupe más en estar bien informado sobre su campo de acción y, autónomamente, se vuelva más competente en el ejercicio de su actividad.

Para preparar mejor a los decentes y, que en consecuencia, logren mejor desempeño, se requiere trabajar con disciplina y esmero en la transformación del modelo pedagógico dominante en las universidades encargadas de formar docentes, trabajo que deberá ser una construcción colectiva entre los docentes 
directivos de la facultad de educación de las distintas universidades; para lo cual es muy necesario que haya una interconexión curricular y metodológica entre las universidades y los colegios.

$\mathrm{Al}$ respecto, De Zubiría (2018) manifiesta su posición sobre el desempeño docente y considera necesario que desde el mismo MEN, se realicen campañas de selección de los mejores docentes en todos los municipios del país a fin de conformar equipos de calidad que, llevados a diferentes colegios, acompañen los procesos de innovación que en ellos se están llevando a cabo; equipos de docentes que se comprometan a reunirse periódicamente para impulsar procesos de formación in situ; nuevas formas y maneras éstas que han demostrado dar resultados favorables y sin las cuales el autor considera es imposible alcanzar el mejor desempeño.

Al respecto, Stenhouse, (1985), expresa que no basta que toda la comunidad educativa se preocupe por la labor docente, sino que deben ser ellos y cada uno de ellos quienes deben hacer un autoestudio que les lleve a trascender del desarrollo de currículos a la investigación y conocimiento de su propia situación, o labor, a fin de lograr en la docencia una realización plena de sus facultades. Dicho de otro modo, el docente debe convertirse en un autodidacta.

En este sentido, Arreola (1994) expresa, "no hay novedad ni reforma si no nos convertimos en verdaderos autodidactas". (p. 33) Expresión que permite aseverar que lo que ofrecen las instituciones de formación docente no es, ni nunca será, suficiente para que el docente desempeñe un buen papel en el aula.

En el mismo orden de ideas, Imbernón (1994), menciona que la formación del profesorado es reconocida cómo área clave que coadyuva a elevar la calidad de la educación, por lo que las instituciones que tienen bajo su responsabilidad formar maestros, deben emplear, en sus planes de formación, estrategias innovadoras que vayan acorde a los resultados de investigaciones psicoeducativas serias que permitan arrojar luces sobre el tipo de docente que el mundo y la sociedad de hoy necesita.

Al respecto, De Zubiría (2017) expone:

...las universidades deben repensar su naturaleza y su función pedagógica y social. Requerimos que ellas analicen a profundidad el modelo pedagógico, hoy centrado en la palabra del docente y en la vieja y tradicional idea de que a la universidad se debería ir a aprender informaciones y algunos conocimientos, pero no a hacerle preguntas a la vida y a transformar las maneras de pensar y valorar. Estudiar -como decía Freire-, "no es un acto de consumir ideas, sino de crearlas y recrearlas". Pero ello, muy poco se aborda en el actual sistema educativo. (p. 23)

La didáctica permite mejorar las prácticas educativas, por cuanto ella proporciona al docente métodos, técnicas y estrategias para facilitar la adquisición de los conocimientos y el desarrollo de habilidades y destrezas. "Esta es una disciplina pedagógica aplicada porque está comprometida con los problemas prácticos que atañen al proceso de enseñanza - aprendizaje y al desarrollo profesional de los docentes" (Celi, 2010, p. 44).

En el caso específico de este estudio, referido al proceso de enseñanza en la básica primaria, se observa con preocupación la poca trascendencia que se otorgan en las escuelas de Colombia al área de la educación física, sin tener en cuenta que la misma es de importancia primordial para el desarrollo de 
las capacidades socio-motrices de los niños y adolescentes.

Al respecto en Colombia, la ley 115 de 1994 (Ley General de Educación), plantea que la educación física tiene como objetivo primordial:

...La valoración de la higiene y la salud del propio cuerpo y la formación para la protección de la naturaleza y el ambiente; el conocimiento y ejercitación del cuerpo, la participación y organización juvenil y la utilización adecuada del tiempo libre... (s/n)

Este objetivo es alcanzable sólo mediante la práctica de la actividad física, la recreación y los deportes, adecuados a la edad, ya que de esta manera se conduce al individuo a un desarrollo físico y armónico.

Hace alrededor de doscientos años la buena forma física por si misma tenía poca importancia. Los escritores contaban las excelencias del montañismo y el pasear, porque constituían pasatiempos agradables que ponían al hombre en contacto con la naturaleza y no porque pudiesen prevenir enfermedades corporales. El ejercicio era además algo inminentemente militar: la equitación, el tiro y la esgrima eran habilidades prácticas, e incluso la natación y el remo tenían su utilidad. Para las mujeres solo se consideraba adecuado el ejercicio suave y la idea de hacerlo, para estar en forma, no dejaba de expresar cierto mal gusto.

Es a principios del siglo XIX, cuando en Europa, en los colegios privados para chicos comienzan a aparecer los deportes organizados, más como diversión que como ejercicio para mantenerse en forma. La puesta en boga del adagio griego de "mente sana en cuerpo sano" da un sentido moral al atletismo, pero todavía no un sentido médico. Y así mientras los jóvenes privilegiados disfrutaban con los deportes que consideraban elegantes, en las escuelas normales o elementales para chicos se introdujo la educación física en forma de instrucción militar y de gimnasia.

En los colegios femeninos no fue sino hasta finales del siglo pasado cuando se introduce la educación física, como asignatura obligatoria. En los EEUU, el doctor Sargent de la universidad de Harvard elabora un plan para crear el físico ideal, un programa complejo y detallado de ejercicios destinados a conformar el cuerpo de acuerdo con unas medidas "ideales" lo que constituyó el primer paso hacia el reconocimiento de que la buena forma física era deseable por sí misma. Más tarde en 1921 Charles Atlas "el hombre más perfectamente desarrollado del mundo" introdujo su famosa "tensión dinámica".

En los años 50, las cifras de muertes por enfermedades cardíacas alcanzan proporciones epidémicas en Europa y en EEUU. Los médicos sabían que la causa principal estaba en la combinación del exceso de comida y la inactividad. Las encuestas permitieron dilucidar la respuesta a la interrogante ¿Por qué hay gente que ignora el consejo de hacer más ejercicio? lo que permitió constatar la confusión existente en lo referente al conocimiento de las necesidades del organismo.

Es así como históricamente la incorporación de la asignatura de educación física en los currículos escolares estuvo inspirada en la concepción platónica de concebir el cuerpo como una maquina en movimiento. De esta forma expresa Contreras (1998), la educación física inicia el interés del cuidado del cuerpo y el mantenimiento de la salud., lo cual según este autor permite centrar su fin en un orden higiénico que la convierte en una especie de medicina preventiva para el mejoramiento de la salud. 
Consecuentemente, la educación física es una parte fundamental en el proceso educativo de toda persona, centrado en el movimiento corporal con el fin de lograr un afianzamiento en las capacidades físicas, cognitivas y afectivas del individuo, especialmente en los años de la niñez.

La educación física aparece en la formación integral de todas las escuelas, tanto primarias como secundarias, de la mayoría de los países del mundo, como un área obligatoria, por la importancia que tiene para la salud, para la formación afectiva y para la relación del niño con sus pares. La articulación de la educación física con las otras disciplinas de aprendizaje está clara, pero generalmente se destaca de ellas por su tendencia a representar para los jóvenes una experiencia muy grata, por su vínculo con lo lúdico y lo recreativo.

En el sistema educativo colombiano, la educación física adquiere una importancia especial al ser considerada la escuela no como un lugar en el que sólo se desarrolla el conocimiento formal de los niños, sino también el lugar donde se transmiten valores, habilidades y competencias relacionadas con el cuerpo y con la actividad motriz, sin la cual se considera difícil perfeccionar los aspectos cognitivos ya que la salud física equivale a salud mental, es decir, la agilidad mental se mueve en paralelo con la agilidad corporal.

Al igual que en las demás áreas del conocimiento, la didáctica de la educación física requiere de una buena organización curricular. Esta organización implica que el profesor, en función de la lúdica, la recreación y el deporte, se responsabilice de la planificación, innovación y creación de situaciones de aprendizaje útiles y necesarias para alcanzar los objetivos deseados.

Sin embargo, en muchas instituciones educativas del país, se observa que los docentes que imparten la asignatura de educación física presentan deficiencias en cuanto a la formación pedagógica y didáctica necesarias para su enseñanza, en sus clases solo desarrollan el aspecto lúdico o deportivo de la educación física y olvidan los aspectos referidos al desarrollo de destrezas y habilidades de la parte psicomotriz.

Igualmente, se observa con preocupación en muchos países y en especial en Colombia, contexto geográfico de este estudio, que en el nivel de primaria esta asignatura es dictada dentro del bloque de materias de los maestros generales; es decir, el maestro monógrado en la primaria es el encargado de desarrollar los contenidos de educación física y por ende el facilitador del logro de las competencias presentes en las disposiciones legales para tal fin.

En efecto, estos docentes desconocen que la didáctica de la educación física conlleva a una actividad de internalización de conocimientos: se espera una reflexión sobre el sentido y los efectos de la actividad motriz, y de los hábitos saludables practicados con regularidad. La educación física persigue la tarea de que los niños incorporen el hábito de mover el cuerpo, para lo cual deben conocerlo muy bien; esto les capacita a identificar la ubicación corporal de cada uno de los más importantes músculos del cuerpo.

En razón de lo planteado, se requiere que el maestro general que maneja la primaria, tenga claro cuáles son los métodos y técnicas didácticas a utilizar en la enseñanza de los contenidos de la educación física, así como el conocimiento sobre los aspectos disciplinares de la asignatura; tal y como lo confirma Torres y Girón (2009), cuando dice que la didáctica se encarga del estudio de todos los principios y técnicas válidas para la enseñanza de cualquier materia o disciplina. Es decir, el docente debe conocer la didáctica a utilizar para los contenidos de educación física. 
Según Torres y Girón (2009), didáctica es hacer que los alumnos aprendan la asignatura de la mejor manera posible, al nivel de su capacidad actual, dentro de las condiciones reales que la enseñanza desarrolla, aprovechando inteligentemente el tiempo, las circunstancias y las posibilidades materiales y culturales que se presentan en la localidad donde se ubica la escuela.

Explica el autor que la didáctica estudia el problema de la enseñanza de modo general, sin las especificaciones que varían de una disciplina a otra. Procura ver la enseñanza como un todo, estudiándola en sus condiciones más generales, con el fin de iniciar procedimientos aplicables en todas las disciplinas y que den mayor eficiencia a lo que se enseña.

Por su parte, Vasco (1990 c.p. González y Malagónlez, 2015), expresa que la didáctica está referida a la reflexión de los diferentes aspectos de las relaciones del docente con sus alumnos, así como de su entorno relacionado con la enseñanza.

En el estudio de la didáctica son muchas las discusiones que se generan, por cuanto siempre se ha cuestionado si el conocimiento del docente debe centrarse solo en los aspectos pedagógicos, (didáctica general) o debe incluir los conocimientos disciplinares (didáctica especial), de esta manera algunos autores plantean lo llamado didáctica especial, referida según Contreras (1998) a la aplicación de la metodología de la didáctica general a un concreto campo disciplinar.

En el caso de la asignatura de educación física, Sánchez Bañuelos (2001 c.p. Carosio 2001) expresa que la didáctica específica de la educación física y el deporte deben adaptarse al desarrollo de una actividad de enseñanza en la que el movimiento corporal y el esfuerzo físico constituyan los contenidos.

De esta manera la didáctica se conforma de actividades o procesos que el docente realiza en la enseñanza. En el caso específico de la educación física, según Echeverria y Morfin (2002), los objetivos, en cuanto al aprendizaje de cada clase, deben tener una secuencia a fin de que se puedan seleccionar adecuadamente los contenidos que servirán de base para proyectar el desarrollo de los estudiantes.

Esto implica, según Sánchez (1992 c. p. Campos 2005), que la enseñanza de la educación física tiene como finalidad la integración del deporte, la recreación, el juego y la adquisición de las habilidades motrices básicas como herramientas que mejoran los procesos de enseñanza aprendizaje desde la edad escolar.

En este sentido, Echeverria y Morfin (2002), consideran que en el proceso de enseñanza de la educación física es importante la correcta distribución del contenido y, las acciones del profesor y el alumno en una sucesión correcta determinada por los métodos y procedimientos de la enseñanza, donde existe una estrecha relación entre objetivo, contenido, método y organización que determina la metodología de la enseñanza de los diferentes aspectos que la componen (juego-gimnasiadeporte).

De esta manera las actividades o procesos que el docente realiza en la enseñanza, conforman la didáctica. En el caso específico de la educación física, expresan Echeverria y Morfin (2002), que los objetivos de cada clase deben tener una secuencia en cuanto a su aprendizaje a fin de que se puedan seleccionar adecuadamente los contenidos que servirán de base para proyectar el desarrollo de los estudiantes.

Esto implica, según Sánchez (1992 c. p. Campos 2005), que en la enseñanza de la educación física se deben integrar el deporte, la recreación, el juego y la adquisición de habilidades motrices básicas como contenidos

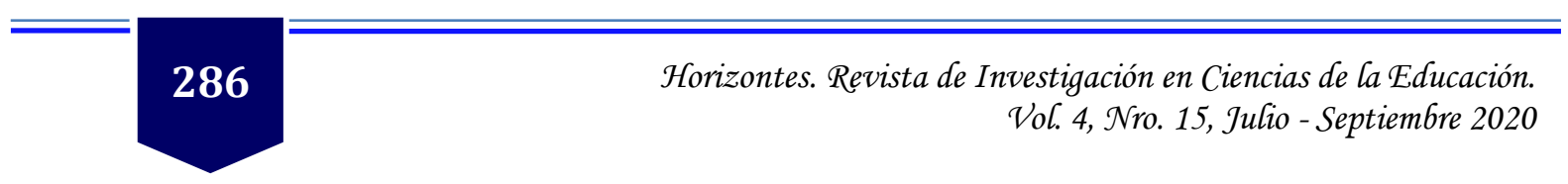


que mejoran los procesos de enseñanzaaprendizaje y proporcionan a los estudiantes herramientas teóricas y conceptuales necesarias que les permite concebir el conocimiento como algo que ofrece la oportunidad de crear y reconstruir, desde la edad escolar, sus propios significados.

Es así como, según Echeverria y Morfin (2002), en la enseñanza de la educación física el docente debe utilizar métodos $\mathrm{y}$ procedimientos didácticos adecuados que permitan un tránsito entre los diferentes niveles de asimilación de manera efectiva, para lo cual su formación es de vital importancia.

Para Gorodokin, (2005 c.p. Feo 2011), la formación docente es el proceso en el que se articulan experiencias de enseñanza y de aprendizaje orientadas a la configuración de sujetos docentes/enseñantes; donde la práctica docente se concibe en un doble sentido: como práctica de enseñanza, propia de cualquier proceso formativo y como apropiación de la profesión docente, cómo iniciarse, perfeccionarse o actualizarse en la práctica de enseñar.

Para Arenas y Fernández (2009), se entiende por formación docente, el conjunto de cursos o eventos académicos con validez oficial, que tienen por objeto habilitar la práctica y/o actualizar a quienes realizan funciones de docencia, en las teorías, procedimientos $\mathrm{y}$ técnicas para impartir la enseñanza, lo cual tiene relación directa con la calidad de la educación en función del mejor desempeño que se tiene como resultado

En el caso específico de los procesos de formación pedagógica del docente de educación física, Sánchez (1992 c. p. Campos 2005), expresa que éstos tienen como finalidad permitir una mejor utilización de los recursos didácticos para implementar las clases y poder despertar en los estudiantes una mayor motivación en sus procesos de formación.

\section{CONCLUSIONES}

En función de todo lo antes expuesto, se considera que los docentes que se desempeñan en las escuelas básicas primaria en Colombia en el dictado de la asignatura educación física, necesitan formación específica complementaria que les capacite y habilite en los diferentes momentos del proceso enseñanza-aprendizaje de la educación física, que como en cualquier otra disciplina requiere de competencias docentes en el manejo de la planificación, la facilitación y la evaluación de los contenidos a transmitir.

Para cada uno de los mencionados momentos es de suma importancia el conocimiento de los contenidos, tal como lo expresa Contreras (1998), cuando expresa que los conocimientos de contenidos del currículo son los referidos a cómo enseñar una determinada asignatura y los de contenidos pedagógicos se dan en relación al cómo enseñar los contenidos propios de la materia. Es decir, se refiere a los métodos y técnicas didácticas específicas utilizadas por el docente.

Con la finalidad de estimular el desarrollo de competencias en los educandos, las competencias docentes guían la práctica docente, es decir, ejercen influencia en la manera de presentar los contenidos, en el diseño de los objetivos, las maneras de transmitir la información, en el cómo dar incentivo a los estudiantes por el estudio, en la evaluación de los aprendizajes; entre otras de sus funciones.

Es así como se demanda al docente de vanguardia la capacidad de reflexión crítica, dinamismo, modificación y/o adaptación de sus prácticas, intercambio con sus pares, uso de la tecnología, liderazgo en la gestión institucional, puesta en práctica de principios como la subordinación de intereses individuales al colectivo, el estímulo y la 
responsabilidad, entre otros; lo cual es una responsabilidad activa de auto reflexión en los docentes acerca de su papel protagónico en la formación de las sociedades del futuro.

En este sentido, es pertinente recordar ciertas características para "un docente ideal" planteadas por Torres y Torres (1999), cuando destacan que "el docente deseado es un profesional competente, agente de cambio, practicante reflexivo, profesor investigador, intelectual crítico y transformador" (p. 2). Igualmente, establecen que este docente requiere dominar contenidos y pedagogías propias de su ámbito de enseñanza, provocar y facilitar aprendizajes basados en el diálogo, la vinculación de la teoría y la práctica, la interdisciplinariedad, la diversidad y el trabajo en equipo; comprender la cultura y las realidades locales; investigar, como modo y actitud permanente de aprendizaje; trabajar y aprender en equipo. ¡Un gran desafío para la docencia siglo XXI!

\section{REFERENCIAS}

Arenas y Fernández (2009) Formación pedagógica docente y desempeño académico de alumnos en la facultad de Ciencias Administrativas de la UABC educación superior Rev. Educ. Sup vol.38 no.150 México abr/jun 2009

Arreola (1994) La palabra educación en la obra de Juan José Arreola. Universidad Nacional de Colombia

Batista, L.; (2006). Educación y desarrollo humano. Costa Rica. http://www.binasss. sa.cr/revistas/enfermeria/v27n1/7.pdf

Campos, A. (2005). Mapas Conceptuales, Mapas Mentales y Otras Formas de Representación del Conocimiento

Carosio, M. (2001) La didáctica de la Educación Física: cuando el conocimiento se trata de "especial". ¿Una didáctica especial para un profesor especial? Revista Digital - Buenos Aires - Año 7 - $\mathrm{N}^{\circ}$ 36 www.efdeportes.com/efd36/ didact.htm
Castillo, Mario; Gamboa, Ronny; (2012). Desafíos de la educación en la sociedad actual. Costa Rica

Celi (2010) ESTRATEGIAS DE ENSEÑANZA Investigaciones sobre didáctica en instituciones educativas de la ciudad de Pasto. Colombia

Contreras, O. (1998) Didáctica de la Educación Física. Un enfoque constructivista. Publicaciones Inde. Barcelona España

Contreras, O (2000) El profesor de Educación Física: antecedentes y paradigmas dominantes en su formación. Actas del XVIII congreso nacional de Educación física. Ediciones de la universidad de Castilla la Mancha, Cuenca. España

Constitución Política y la Ley General de Educación (Ley 115), Ministerio educación de Colombia, (2014). Formación docente, un aspecto clave para la calidad de la educación en Colombia

De Zubiría, J (2018) Hacia un acuerdo nacional para mejorar la calidad de la educación básica y media en Colombia. Bogotá. Colombia

Echeverría y Morfin (2002) Dirección del proceso de enseñanza-aprendizaje en la clase de Educación Física. Revista Digital Buenos Aires - Año 8 - $\mathrm{N}^{\circ} 50$. www.efdeportes.com/efd50/claseef.htm

Feo, (2011). Una mirada estratégica a la formación docente de calidad. Venezuela

González, H. y Malagónlez, R. (2015). Elementos para pensar la formación pedagógica y didáctica de los profesores en la universidad. Colombia. Appl. Linguist. J., 17(2), pp. 290-301. Documento en línea disponible en: http://www.scielo.org.co/pdf/calj/v17n2 /v17n2a09.pdf

Henz (1976) La promoción y desarrollo de los valores sociales, objetivo prioritario de la formación social: un enfoque interdisciplinario Vol. 4, № 2 (Nueva Serie), 2004: 13-37

Hurtado (2010). Metodología de la Investigación. Guía para la comprensión holística de la ciencia. Ediciones Quirón. Caracas, Venezuela 
Imbernón, (1994). La formación y el desarrollo profesional del profesorado en España y Latinoamérica Revista Electrónica Sinéctica, núm. 41, Instituto Tecnológico y de Estudios Superiores de Occidente Jalisco, México

Ministerio de Educación Nacional (1994). Ley General de Educación 115. República de Colombia

Stenhouse, Lawrence (1985) Investigación y desarrollo del Curriculum, Morata: Madrid. pp. 194-221

Torres y Girón (2009) Didáctica General https://ceccsica.info/sites/default/files/c ontent/Volumen_09.pdf

Torres y Torres, (1999) Dirección Estratégica un enfoque práctico principios y aplicaciones de la gestión del rendimiento. Ediciones Díaz de Santos Casa del libro.

Unesco (2011). La Unesco y la educación.
[Página web en línea]. Consultado el 10 de noviembre de 2016 en: http://unesdoc. unesco.org/images/0021/002127/21271 5 s.pdf

Unesco (2013) Situación Educativa de América Latina y el Caribe: Hacia la educación de calidad para todos al 2015 Publicado por la Oficina Regional de Educación para América Latina y el Caribe (OREALC/UNESCO Santiago)

Unesco (2014) Estrategia de Educación de la UNESCO2014-2021 7, place de Fontenoy, 75352 París 07 SP, Francia

Unesco (2016). Recomendaciones de política educativa en América Latina en base al TRECE. Santiago de Chile

Valenzuela, Mónica; (2010). Importancia de la educación en la actualidad. Guía con las bases metodológicas e investigadoras para una mejora de la educación. España 